\title{
Two-Dimensional Radar Imaging of Flowing Avalanches
}

\author{
Matthew Ash", ${ }^{\mathrm{a}, *}$ Paul V. Brennan ${ }^{\mathrm{a}}$, Chris J. Keylock ${ }^{\mathrm{b}}$, Nathalie M. Vriend ${ }^{\mathrm{c}}$, \\ Jim N. McElwaine ${ }^{\mathrm{d}, \mathrm{e}}$, Betty Sovilla ${ }^{\mathrm{e}}$ \\ ${ }^{a}$ Department of Electronic and Electrical Engineering, University College London, \\ London, UK. \\ ${ }^{b}$ Department of Civil and Structural Engineering, University of Sheffield, Sheffield, UK. \\ ${ }^{c}$ Department of Applied Mathematics and Theorectical Physics, University of Cambridge, \\ Cambridge, UK. \\ ${ }^{d}$ Department of Earth Sciences, Durham University, Durham, UK. \\ ${ }^{e}$ WSL Swiss Federal Institute for Snow and Avalanche Research, SLF, Davos, \\ Switzerland.
}

\begin{abstract}
Radar has emerged as an important tool in avalanche research. However, existing radar sensors suffer from coarse range resolution capabilities. This limits the usefulness of the data they collect in validating models of avalanche dynamics. This paper details the development of a frequency modulated continuous wave, phased array radar, and its associated signal processing, for non-invasive measurements of entire avalanche events. The radar outperforms existing avalanche radar sensors in terms of range resolution, and it provides cross-range resolution using a phased array receiver. The radar has been operating at the Vallée de la Sionne avalanche test site in Switzerland since the 2010 winter season. It has successfully gathered measurements of entire natural avalanche events. In this paper we show two-dimensional radar images of a naturally occurring avalanche, the first of their kind, which re-
\end{abstract}

\footnotetext{
*Corresponding author. Tel.: +44 2076790153.

Email address: m.ash@ucl.ac.uk (Matthew Ash)
} 
veal movements of layers or particles of the flowing avalanche in unparalelled detail. Furthermore, the potential of the measured data is shown with tracking of avalanche fronts in two spatial dimensions. This marks an important step towards providing a library of high-quality avalanche measurements to improve our knowledge of avalanche dynamics.

Keywords: Radar, FMCW, phased array, avalanche dynamics, imaging

1 1. Introduction

$2 \quad$ Avalanches pose a significant threat to human life and settlements. Haz3 ard and risk assessments of avalanche prone regions are typically based on 4 the statistical analysis of observed avalanche run-out distances (Keylock 5 et al. 1999), or more commonly, combine knowledge of extreme snow depths (Blanchet et al., 2009) with a dynamics model to assess run-out properties 7 (Eckert et al., 2010) or potential damage and vulnerability (Keylock and 8 Barbolini, 2001; Bertrand et al., 2010). Given the importance of these nu9 merical models for accurate risk assessments, it is not surprising that there 10 have been concerted efforts to develop physically meaningful flow laws for 11 inclusion in numerical codes (Eglit, 1968; Norem et al., 1986; Nishimura and 12 Maeno, 1988; Salm, 1993; Dent et al., 1998; Gray and Tai, 1998; Bouchet 13 et al., 2003; Kern et al., 2004). Such research has also examined the role 14 of entrainment processes in the dynamic behaviour Gauer and Issler, 2004; 15 Naaim et al., 2004) and there have also been attempts to benchmark models against one another for recorded events (Barbolini et al., 2000; Issler et al., 2005). However, the lack of high-quality data still means that there is still 18 significant uncertainty in the relevant physics for flowing snow, making model 
validation problematic.

Radar has emerged as an important tool in avalanche research for obtaining avalanche measurements. It has been exploited for gathering velocity measurements of entire avalanche flows (Gubler et al., 1986; Schreiber, 2001; Rammer et al. 2007), and localised erosion and deposition measurements (Gubler and Hiller, 1984). The use of these measurement instruments has led to great improvements in our knowledge of avalanche behaviour over the last couple of decades, with attempts made to determine rheological parameters from such data (Ancey and Meunier, 2004). However, these measurements suffer from weaknesses in certain respects, in particular regarding range resolution. Consequently, the drive for developing a better understanding of avalanche dynamics is hindered somewhat by a lack of high-quality data.

Localised radar measurements clearly do not provide an accurate picture of the entire avalanche. Non-invasive measurements of entire avalanche flows have been taken with pulse-Doppler radar to produce localised velocity maps of the avalanche using range gating. Indeed, the usefulness of these measurements has been demonstrated with the recording of the spread of velocities along an avalanche, showing that velocities decrease rapidly behind the avalanche front, which has the maximum speed (Gauer et al., 2007). However, the finest range gates before the development of the instrument discussed in this paper were $25 \mathrm{~m}$. This limited range resolution means that localisation of sub-components of the avalanche is not possible. Furthermore, these radar are single channel systems, limiting measurements to a single (range) dimension, hence missing the lateral dynamics of the avalanche.

3 In this paper we describe a novel frequency modulated continuous wave 
(FMCW) phased array radar capable of taking non-invasive measurements of entire avalanche events with a range resolution that greatly improves upon existing radar instruments and provides cross-range resolution for the first time. We will provide an in-depth description of the radar and antenna array design, and an analysis of the system performance. We also detail the signal processing steps required to produce avalanche images from the radar measurement data. Finally, we will report some measurements of avalanches as recorded during a winter season, including two-dimensional radar images of a flowing avalanche for the first time.

\section{Radar System Design}

\subsection{Microwave Characteristics of Snow}

The radar system operates in the microwave electromagnetic spectrum and its design started with a brief analysis of the microwave characteristics of snow so that a radar link budget could be formulated. An avalanche, and the regions comprising an avalanche, can be characterised by their snow/ice density, water density and air density. In the interest of formulating a link budget, it is good practice to design for the worst-case scenario. In this case, that scenario is the one which provides the lowest backscatter coefficient, $\sigma^{\circ}$, a dimensionless quantity that describes the average effectiveness of a surface to scatter radiation upon it. In this paper, we are interested in measuring the underlying dynamics of the dense core region of an avalanche. This region tends to have the highest snow and water density within the avalanche. The density of the water content defines whether the avalanche is dry or wet. In this application, the radar is designed to measure the dynamics of dry snow 
74

avalanches in the first instance. For a dry snow medium, the contribution of the imaginary part to the complex dielectric constant is negligible. It is generally agreed that the real part of the dielectric constant of dry snow is a function of snow density. The suggested practical model for the real part of the relative dielectric constant of dry snow is (Tiuri et al., 1984; Hallikainen et al., 1986):

$$
\epsilon_{r} \approx 1+2 \rho_{d s}
$$
where $\rho_{d s}$ is the bulk dry snow density relative to density of water. This model is suitable for $\rho_{d s} \leq 0.5$, beyond which the error increases rapidly. The dielectric constant, $\epsilon_{r}$, of a dry snow medium is therefore approximately 1.2 assuming that the dense core region of the dry snow avalanche has a density of $100 \mathrm{~kg} \mathrm{~m}^{-3}$ and that water has a density of $1000 \mathrm{~kg} \mathrm{~m}^{-3}$. This approximation lies somewhere between the fluidized and dense region density (McClung and Schaerer, 2006; Schaer and Issler, 2001) and does not consider the variation in snow temperature and thus density along the avalanche path Steinkogler et al., 2014). From this figure, we can calculate the backscatter coefficient using an equation derived from the reflection coefficient of a transmission line (Pozar, 2012):

$$
\begin{aligned}
\Gamma & =\frac{Z_{1}-Z_{2}}{Z_{1}+Z_{2}}=\frac{\sqrt{\epsilon_{r}}-1}{\sqrt{\epsilon_{r}}+1} \\
\sigma^{\circ} & =|\Gamma|^{2}
\end{aligned}
$$


where $Z_{1}$ is the wave impedance of free space and $Z_{2}$ is the wave impedance of the target snowcover. This gives an approximate backscatter coefficient of 0.002 at a grazing angle (the angle between the target surface and the incident ray) of $90^{\circ}$. For a particular radar system, the backscatter coefficient is a function of snowcover characteristics, grazing angle and surface roughness. Predicting and modelling some of these parameters is not a trivial task so for the link budget calculations in this paper we have assumed a grazing angle of $7^{\circ}$, based on the worst-case geometry in this scenario, and a rough surface (the standard deviation of the surface height during a flowing avalanche is likely to violate the Fraunhofer criterion (Ulaby et al., 1982)). For a rough surface the variation in backscatter coefficient with grazing angle is less pronounced relative to a smooth surface (Ulaby et al., 1982), hence the link budget calculations in this paper we have not considered the angular dependence of the backscatter coefficient.

This system is primarily designed to provide measurements of snow movement and so the absolute value of the reflected signal is not of great importance. However, clearly it is necessary to have an understanding of the composition of these movements. The penetration depth of the radar signal is also dependent on the snow density and its water content (Ulaby et al. 1986). Our radar operates at C-band, at which the radar signal penetration depth for dry snow has been shown to be up to $10 \mathrm{~m}$ (Rignot et al. 2001). Hence, the backscattered radar signal is a superposition of reflections from the snow-air interface, the below-surface snow volume (which is comprised of multiple ice layers), and potentially the snow-ground interface. The re- 
sults in the latter sections of the this paper will show the ability to see these avalanche movements following processing of the recorded radar data.

\subsection{Radar Considerations}

The new radar, Geodar, operates at $5.3 \mathrm{GHz}$ (C-band), $5.7 \mathrm{~cm}$ free-space wavelength, in order to illuminate the blocks of snow comprising the dense core region of an avalanche, which are assumed to be in the order of centimetres to metres in size. The radar is of frequency modulated continuous wave type, meaning it continuously transmits a radar signal that is frequency modulated. In this case, the radar employs a linear frequency ramp (chirp) as the radar signal, which is described by:

$$
y_{t}=a_{t} \cos \left(2 \pi f_{o} t+\pi \alpha t^{2}\right)
$$

where $a_{t}$ is the signal amplitude, $f_{o}$ is the radar operating frequency, $\alpha$ is the chirp rate (ratio of chirp sweep bandwidth $B$ and chirp period $T$ ). The range resolution of such a radar signal is given by the well-known expression (Skolnik, 2001),

$$
\Delta R=\frac{c}{2 B}
$$

where $c$ is the signal propagation speed (assumed in calculations to be the approximate speed of light in a vacuum of $3 \cdot 10^{8} \mathrm{~m} / \mathrm{s}$ ). On reception, following two-way propagation, the radar signal is mixed with a portion of the 
transmitted signal in a process known as deramping. This generates a beat signal whose frequency can be described by (Stove, 1992),

$$
f_{d}=\frac{2 \alpha R}{c} \pm \frac{2 f_{o} v}{c}
$$

where $v$ is the velocity of the target at range $R$ and assuming that the radar signal propagates through free space. The \pm sign is included to indicate the use of triangular modulation in the transmitted radar signal; this will be explained later in this text. It is this beat signal that is recorded by the radar analog-to-digital converter. Spectral analysis of the recorded signal is used to relate the signal to target range.

The maximum frequency sweep bandwidth of the radar is $200 \mathrm{MHz}$ which gives a minimum theoretical range resolution of $0.75 \mathrm{~m}$, a great improvement over existing avalanche radar instruments. The linear chirp period can be varied between $1 \mathrm{~ms}$ and $5 \mathrm{~ms}$. This gives a deramp frequency of between $1333 \mathrm{~Hz} / \mathrm{m}$ and $267 \mathrm{~Hz} / \mathrm{m}$. These parameters demonstrate some of the advantages of using an FMCW radar; namely that one can achieve high range resolution with a modest receiver sampling rate. In this case, the system employs a sampling rate of $2 \mathrm{MSa} / \mathrm{s}$ for each radar channel. This provides a maximum range of $\left(\frac{10^{6}}{267}=\right) 3750 \mathrm{~m}$ to satisfy the Nyquist sampling criterion. A moving target will produce a Doppler frequency of $35 \mathrm{~Hz} / \mathrm{ms}^{-1}$.

The link budget can be calculated using the radar equation modified for a distributed target. The distributed target radar equation can be expressed in the following form (Ulaby et al., 1982): 


$$
P_{r}=\frac{\lambda_{o}^{2}}{(4 \pi)^{3}} \int_{\text {area illuminated }} \frac{P_{t} G_{t} G_{r} \sigma^{o}}{R^{4}} d A
$$

152

where $P_{r}$ is the received power, $\lambda_{o}$ is the operating signal wavelength, $P_{t}$ is the transmitted power, $G_{t}$ is the transmitter antenna gain, and $G_{r}$ is the receiver antenna gain. Using the range resolution and beamwidth, the distributed target radar equation can now be evaluated. The grazing angle will affect the length measured along the surface and therefore the area of interception known as a resolution cell (see Figure 1). If we make reasonable assumptions that the length along the surface does not fill the entire beam, the antenna gain does not vary across the resolution cell, and for a long range application, we can make the following simplification:

$$
P_{r} \approx \frac{P_{t} G_{t} G_{r} \lambda_{o}^{2}}{(4 \pi)^{3} R^{4}} \int_{\text {area illuminated }} \sigma^{o} d A=\frac{P_{t} G_{t} G_{r} \lambda_{o}^{2}}{(4 \pi)^{3} R^{4}}\left[\frac{\Delta R}{\cos \psi} \cdot R \theta_{B, a z}\right]
$$

where $\psi$ is the grazing angle, and $\theta_{B, a z}$ is the azimuthal beamwidth of the transmitter antenna. Hence, the modified distributed target radar equation and signal-to-noise ratio (SNR) for a particular target range in this scenario can be described by:

$$
\begin{aligned}
P_{r} & =\frac{P_{t} G_{t} G_{r} \Delta R \theta_{B, a z} \sigma^{o} \lambda_{o}^{2}}{(4 \pi)^{3} R^{3} \cos \psi} \\
S N R_{o} & =\frac{P_{t} G_{t} G_{r} \Delta R \theta_{B, a z} \sigma^{o} \lambda_{o}^{2}}{(4 \pi)^{3} R^{3} \cos \psi k T_{o} B_{n} F_{n}}
\end{aligned}
$$


with $k T_{o} B_{n} F_{n}$ representing the receiver thermal noise power where $k$ is Boltzmann's constant, $T_{o}$ is the operating temperature in Kelvin, $B_{n}$ is the coherent processing bandwidth $\left(\approx \frac{1}{T}\right)$, and $F_{n}$ is the receiver noise factor.
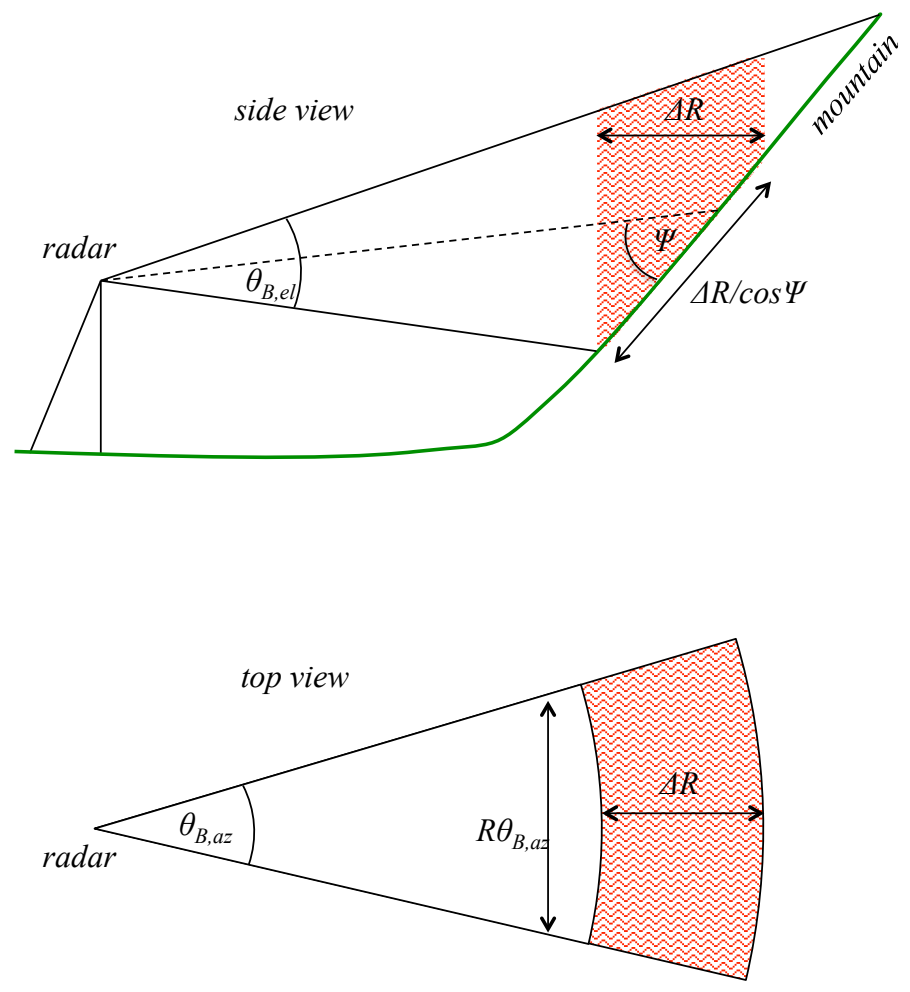

Figure 1: The effect of grazing angle $\psi$ on length measured along a surface and the effect of range on the azimuthal width of resolution cell.

171 It can be seen in Equation (6) that embedded in the deramp frequency is 172 the Doppler frequency indicating a moving target. This equation shows that for a moving target the deramp frequency will be shifted such that the apparent range of the target is changed. This effect is known as range-Doppler cross-coupling and there are ways of mitigating its effect. The Doppler fre- 
quency affects up and down chirps in an equal and opposite way (as indicated by the \pm sign). Hence it is possible to resolve and remove, or exploit, this Doppler frequency by using triangular modulation of the chirp signals, i.e. alternate between transmitting an up chirp and a down chirp, and processing the return signals as pairs (one up and one down). The new radar exploits this technique but also uses a technique of varying the chirp periods in order to build extra redundancy into the data. This aids resolving the Doppler frequency from the deramp frequency during signal processing. A time domain plot of a single frame of the radar signal is shown in Figure 2. Each frame has a period of $20 \mathrm{~ms}$ giving an update rate of 50 frames per second. There are gaps in transmission at the beginning of each frame and between chirps. This is to provide clear markers to aid the process of splitting the receiver data into returns from each of the six chirps. It can be seen that there is also some amplitude tapering applied to the beginning and end of each chirp signal. This is to give a more gentle step change in signal level at these points placing less transient strain on the receiver baseband amplifiers. In addition, there is a short period of tapering in the middle of each chirp, or blanking. This blanking period corresponds to when radar signal sweeps through the baseband frequencies of the receivers. This technique was employed to remove the effect of a non-linearity in the receiver deramping mixer, which causes the radar signal to leak in to the receiver at baseband frequencies. It is also possible to apply this blanking during signal processing of the recorded data. 

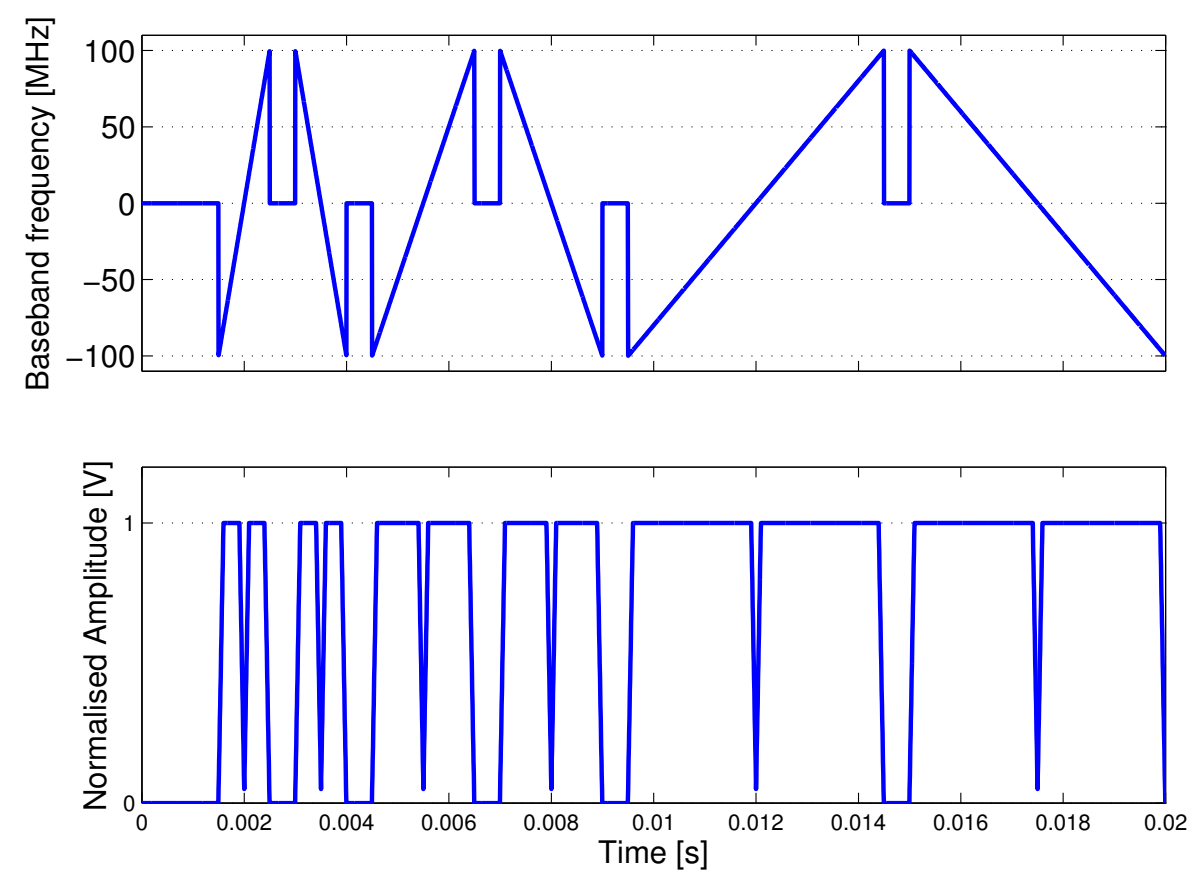

Figure 2: Format of the system frequency modulated signal frames. The total frame duration is $20 \mathrm{~ms}$. 


\subsection{Radar Specifications and Performance}

The radar design is shown as a block diagram in Figure 3, including all of the part numbers of the commercial components used. The FMCW radar signal is generated using an arbitrary waveform generator which is upconverted to the operating frequency in two stages using low phase noise frequency synthesisers (VIDA products inc. Hammerhead range) at $3 \mathrm{GHz}$ and $8.3 \mathrm{GHz}$. The transmit power is adjusted using a digital step attenuator which allows adjustments in $0.5 \mathrm{~dB}$ steps. The transmit signal is heavily filtered using a $5.3 \mathrm{GHz}$ cavity filter with a $200 \mathrm{MHz}$ pass-band. The radar transmit power is $15 \mathrm{~W}$. The transmitter and receiver antennas have a gain of $12 \mathrm{dBi}$ and an azimuth beamwidth of $29^{\circ}$.

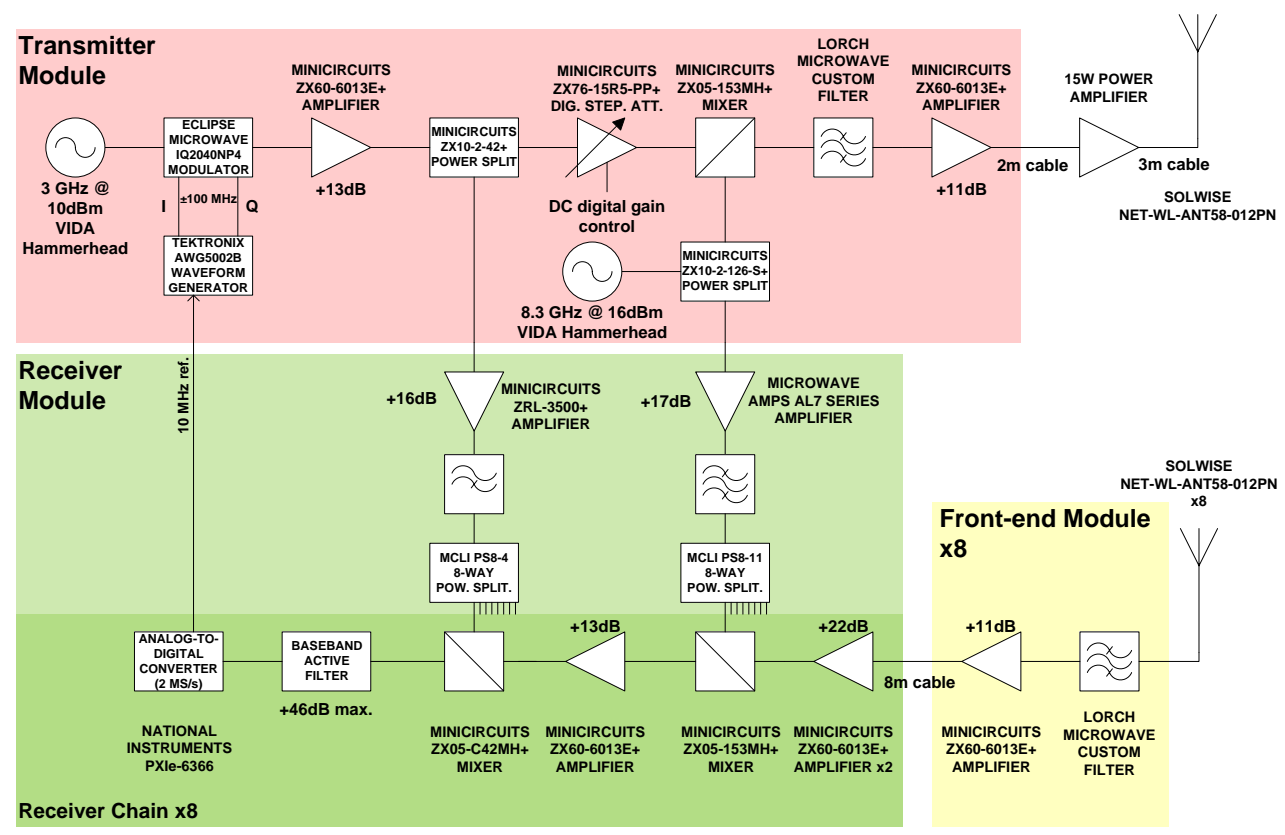

Figure 3: Full system block diagram of the frequency modulated continuous wave radar. 
The receiver comprises eight identical channels. The front-end consists of the same cavity filter used in the transmitter and a low noise amplifier. The incoming FMCW signal is down-converted using a portion $8.3 \mathrm{GHz}$ local oscillator signal and then deramped using the $3 \mathrm{GHz} F \mathrm{FMCW}$ signal from the transmitter. Following deramping, the baseband signal is conditioned with a carefully designed active filter that has a high-pass characteristic to compensate for propagation losses associated with variable range targets. This technique is commonly known as frequency gain control (Stove, 1992). It also implements a low-pass filter that acts as an anti-alias filter before analogue-to-digital conversion.

The noise figure of the receiver was measured across the baseband bandwidth using the gain method (Friis, 1944). First, the system gain over the bandwidth was measured. Then a recording was taken with the receiver input terminated in $50 \Omega$. De-embedding the measured gain and the noise purely due to ambient temperature gives a measurement of the noise figure. The noise figure varies with frequency due to the use of frequency gain control. The minimum noise figure of a typical receiver is $8 \mathrm{~dB}$. From these noise figure measurements we can predict the receiver SNR variation with range for a specified target using Equation (10). Figure 4 shows the SNR variation with range for a snow target modelled with a backscatter coefficient of 0.002 and a $7^{\circ}$ grazing angle calculated using Equation (10). The SNR at the maximum required range $(\approx 2500 \mathrm{~m})$ is over $18 \mathrm{~dB}$ which makes the system suitable for the detection of snow along the entire Vallée de la Sionne avalanche track. 


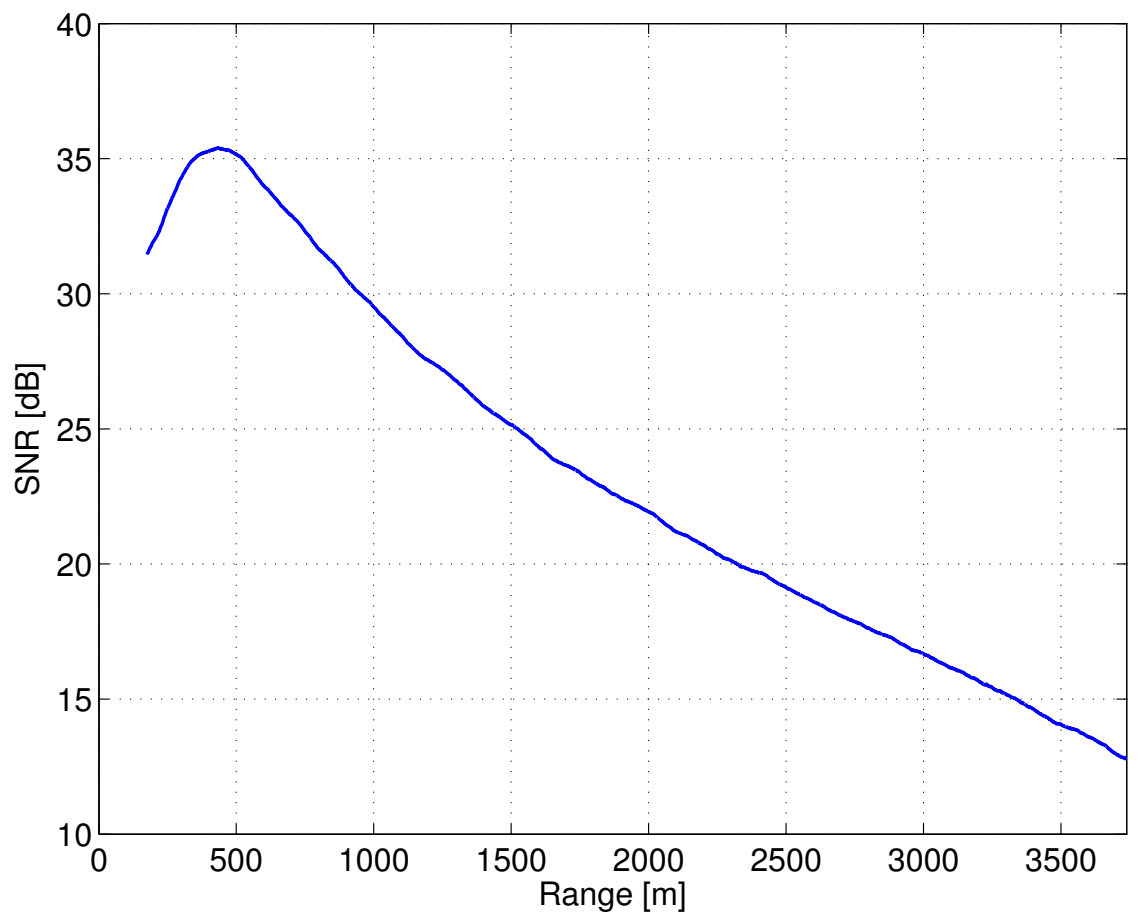

Figure 4: Predicted signal-to-noise ratio (SNR) for $5 \mathrm{~ms}$ up chirp using receiver noise measurements and a modelled target with a backscatter coefficient of 0.002 at $7^{\circ}$ grazing angle. The SNR at the maximum required range $(\approx 2500 \mathrm{~m})$ is over $18 \mathrm{~dB}$ which makes the system suitable for the detection of snow along the entire Vallée de la Sionne avalanche track. 


\subsection{Receiver Antenna Array}

In order to provide cross-range measurements of an avalanche, the new radar employs a receiver array comprising eight receiver antenna elements. The radar is deployed at the avalanche bunker at Vallée de la Sionne (see Section (4) with the receiver antennas mounted on an outside wall facing the avalanche track. In order to make maximum use of the available horizontal baseline, the receiver elements are spread across the width of the bunker wall in a line as shown in Figure 6. The total width of the array is $5.31 \mathrm{~m}$. This indicates an undersampled aperture with a mean inter-element spacing of $14 \lambda$. In order to minimise the effect of grating lobes, the antenna locations were randomised (Steinberg, 1976). This was achieved by numerically generating a set of randomised locations and selecting the best array factor response based on sidelobe level within the required field-of-view (FOV) of $\sin \theta= \pm 0.26$. Figure 6 shows the element locations and the array factor of the final design. The array factor has been simulated using elements with $29^{\circ}$ beamwidth and a cosine field distribution. The typical sidelobe level is around $8 \mathrm{~dB}$ below the mainlobe and the peak sidelobe level is $4.45 \mathrm{~dB}$ below the mainlobe. Inspection of the array factor shows the array half-power beamwidth $\left(\theta_{a}\right)$ is $0.5^{\circ}$ at $0^{\circ}$ pointing direction which equates to a cross-range resolution of $9 \mathrm{~m}$ at $1 \mathrm{~km}$ range. 


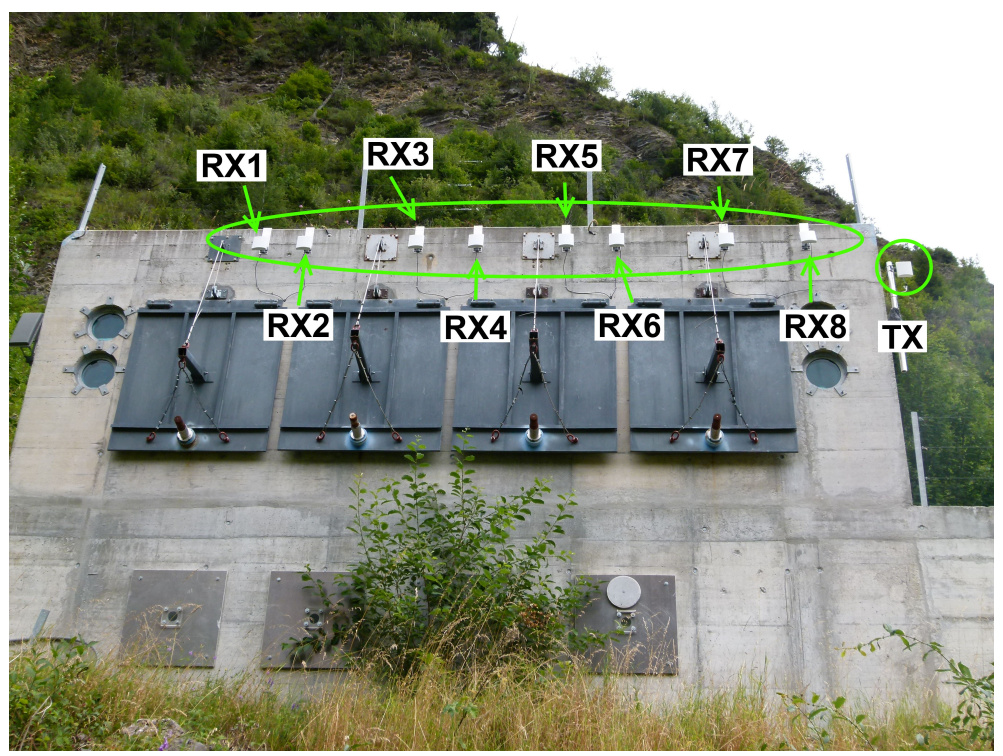

Figure 5: Antenna array setup in Vallée de la Sionne test bunker. The transmitter antenna (TX) is located on the south-facing wall of the shelter pointing towards avalanche track at an angle of $12^{\circ}$ relative to horizontal. The eight receiver antennas (RX1-8) are randomly spaced above the four shelter windows. The receiver antennas are also pointed towards the avalanche track. 


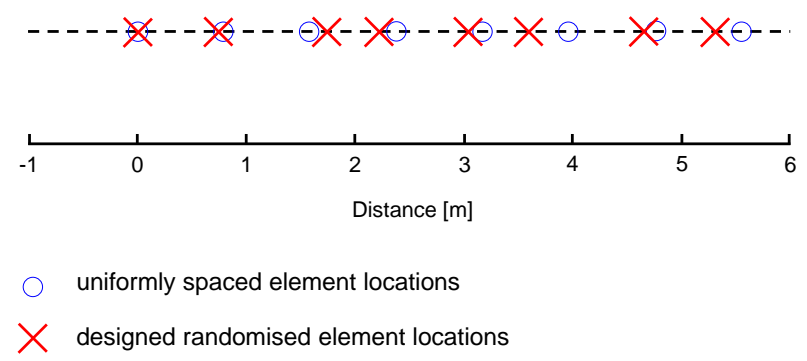

(a)

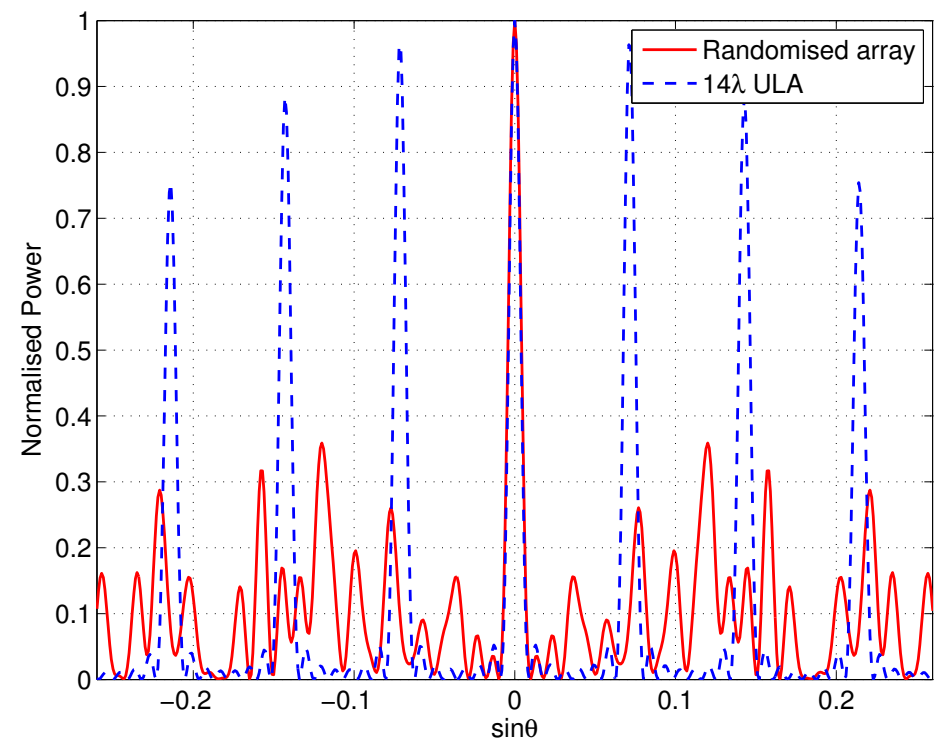

(b)

Figure 6: (a) Diagram of the linear receiver array antenna element positions used in the newly developed avalanche radar. The element locations have been randomised to minimise the effect of sidelobes. (b) Array factor of radar receiver array using directive cosine elements over $\sin \theta= \pm 0.26$ with array factor of uniformly spaced linear array with the same baseline and directive elements. 


\section{Radar Signal Processing}

The radar design presents some interesting signal processing challenges in order to generate 2-D images of the recorded avalanches. This section details the processing steps that were developed to generate these images.

The radar data are recorded by an eight channel data acquisition system which samples at a rate of $2 \mathrm{MSa} / \mathrm{s}$ with 16 -bit resolution. The output of each radar channel is a deramped time domain signal, which requires some spectral analysis in order to extract the range information. However, before this, the signal must be split into the deramped signal associated with each of the six chirp waveforms. This is carried out by cross-correlating the amplitude envelope of the FMCW signal frame (Figure 2) with the recorded time domain data. A correlation peak indicates the beginning of a new waveform frame as a sampling instance. From this, the beginning and end of each of the six chirps can be predicted using the format of the FMCW signal frame. With knowledge of the beginning and end of each chirp, the data are reshaped to form a 3 -D array $A_{i j k}$, where $i=1 \ldots N_{\text {fast-time }} ; j=1 \ldots N_{\text {slow-time }}$; $k=1 \ldots 6$ (chirp number). This process is visualised in Figure 7 for clarity.

Range processing is carried out on each chirp separately in the first instance. Prior to range processing, the individual chirp returns are windowed using a Blackman window to reduce the effect of range-sidelobes (equivalent to reducing the effects of spectral leakage in a discrete Fourier transform (Harris, 1978)). This process degrades the nominal range resolution to $1.3 \mathrm{~m}$. Range processing is achieved by performing fast Fourier transforms (FFTs) along the fast-time axis and relating each frequency bin to a slant range (lineof-sight path length from radar to range bin) using Equation (6) modified to 

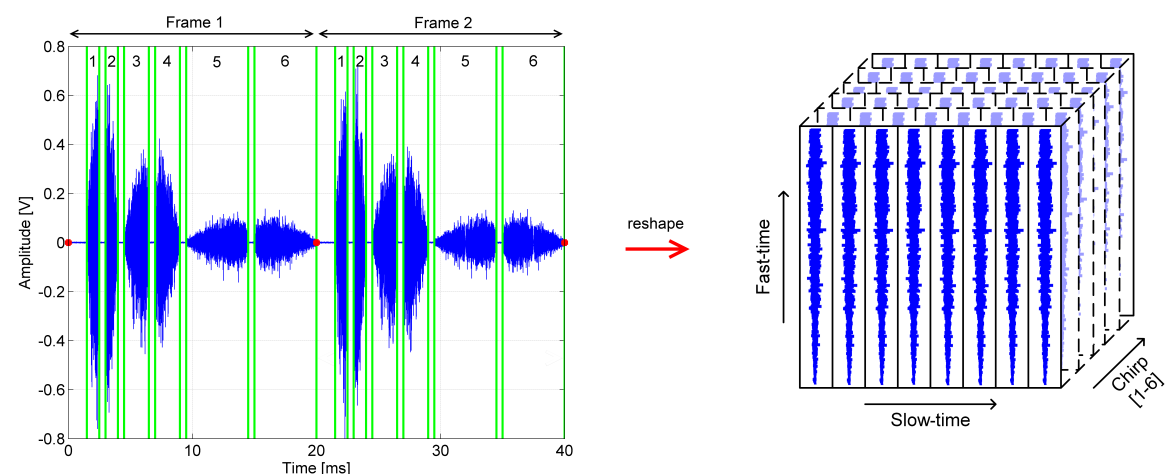

Figure 7: Example time domain data labelled into the returns from each of the six chirps. The data are reshaped into six two-dimensional arrays with columns of short time and rows of fast time.

ignore the Doppler frequency (i.e. $v=0$ ).

The data are prepared for full range plots (such as that shown in Figure 10) by taking the magnitude of the complex Fourier-transformed data and applying a compensation for the losses associated with the two-way propagation of the radar signal. Based on the previously derived radar equation (10), the received signal power is inversely proportional to the cubic range. This drop in signal level with range is in fact over-compensated for in hardware by the baseband active filter. Hence, following range processing, the data are normalised by the measured frequency characteristic of the filter to remove its response. Following this, the data are multiplied by a range dependent factor, $\Omega(R)=R^{3}$, along the fast time (range) axis to give an ideal compensation for propagation losses.

Before generating 2-D images, the receiver array data are calibrated for array distortions. The set up and design of the radar system precludes accurate knowledge of receiver element location as it is difficult to measure 
antenna locations in the field to within a fraction of a wavelength. Additionally, the precise electrical length of each of the receiver chains is not known as measuring them once the radar was installed was not possible with the available equipment. This results in a distortion of the array which must be corrected to obtain focussed images from the radar data. An antenna array such as this can be calibrated by applying phase corrections to the signals impinging on each antenna element. In this system we use Steinberg's radio camera algorithm (Steinberg, 1981) to achieve calibration. The algorithm exploits a stationary scatterer located on the mountain referred to as a dominant scatterer. In early experiments the dominant scatterer used was a natural feature of the mountain, possibly a flat rock face. Indeed, in the data presented in this paper, a natural feature has been used as the dominant scatterer. The scatterer exists in the $1821 \mathrm{~m}$ range bin and is present in all the datasets from the same winter season. In more recent experiments, an active target has been deployed on the mast in the avalanche track. This provides a very high signal-to-noise ratio target that is ideal for the dominant scatterer algorithm. As a consequence, we anticipate higher quality results from the latest experiments.

The first step of the algorithm is to measure the phase of the range bin in which the dominant scatterer exists $\left(\phi_{0, n}\right)$ in each of the $n=1 \ldots 8$ array elements, and in the case of the natural feature these phases are averaged over entire datasets to improve the signal-to-noise ratio. In this case, the phases at $1821 \mathrm{~m}$ were averaged over 5900 frames $(118 \mathrm{~s})$. The phase of the target signal is measured at each antenna element $n$ and can be described by: 


$$
\phi_{0, n}^{\prime}=-k R_{0, n}+\beta_{n}^{\prime}+\delta \phi_{0, n}^{\prime}
$$

319

where $k$ is the wavenumber, $R_{0, n}$ is the path length to the dominant scatterer to element $n, \beta_{n}^{\prime}$ is the measured phase error due to travelling through its receiver system and its associated circuits, and $\delta \phi_{0, n}^{\prime}$ is the phase measurement error due to clutter (unwanted echoes from surrounding features such as foliage, rock faces, etc.). The array origin is assumed to be element 0 and the phase measured at this element $\left(\phi_{0,0}^{\prime}\right)$ is subtracted from the phases measured on all the other array elements $\left(\phi_{0,1-n}^{\prime}\right)$. This normalisation process removes the effect of target distance and isolates the phase due to array distortion effects and clutter. Subtracting the normalised phase from all range bins at each element compensates for array distortions over all ranges. Then, the array is focused on all range bins by compensating for the phase variation with range. The cophased output of the algorithm can be summarised by:

$$
B_{i, n}=A_{i, n} \exp \left[j\left(\phi_{i, n}-\phi_{0, n}+\phi_{0,0}+\frac{k x_{n}^{2}}{2}\left(\frac{1}{R_{i}}-\frac{1}{R_{0}}\right)\right)\right]
$$

where $\phi_{i, n}$ is the phase at range bin $i$ measured by array element $n, k$ is the wavenumber, $x_{n}$ is the distance to element $n$ from the reference element, $R_{i}$ is the range bin, $R_{0}$ is the dominant scatterer range bin, and $j$ indicates the imaginary part. The right hand parts of the equation perform the focusing over all range bins. In this algorithm, the phase error due to clutter $\delta \phi_{0, n}$ has been assumed to be constant over range. This is the main source of error in the algorithm. 
350

Once the array has been calibrated, the process of beamforming can be carried out in order to produce two-dimensional images of the avalanche using the well-known beamforming equation (Steinberg, 1976):

$$
B(\theta)=\sum_{n=1}^{N} W_{n} e^{j k d_{n}\left(\sin \theta-\sin \theta_{0}\right)}
$$

where $W_{n}$ is the amplitude weighting at element $n, d_{n}$ is the distance between element $n$ and the reference element, and $\theta_{0}$ is the pointing direction. Amplitude weighting was used to reduce the close-in spatial sidelobe levels at the expense of a slight degradation in array beamwidth (from $0.5^{\circ}$ to $0.6^{\circ}$ ). A gentle raised cosine taper $(p=0.6)$ was used as the weighting function (Trees, 2002):

$$
\begin{aligned}
W_{n}\left(d_{n}\right) & =p+(1-p) \cos \pi\left(\frac{d_{n}-\frac{L}{2}}{L}\right) \\
& =p+(1-p) \sin \pi\left(\frac{d_{n}}{L}\right)
\end{aligned}
$$

where $L$ is the total width of the array $(5.31 \mathrm{~m})$.

The dynamic range of the background clutter measured at the avalanche track is high. It also has high signal strength. The major clutter components are returns from rock and foliage in the avalanche track. These can be assumed to be stationary throughout the course of a measurement. Hence, they can be removed using a moving target indication (MTI) filter along the slow-time axis of the complex range data. To implement an MTI filter, we use a first order infinite impulse response (IIR) filter of the following form: 


$$
\begin{aligned}
V_{f}\left[n_{f t}\right] & =\beta V_{u}\left[n_{f t}\right]+\xi V_{f}\left[n_{f t}-1\right] \\
\beta & =\frac{\omega_{c} \Delta t}{1+\omega_{c} \Delta t} \\
\xi & =\frac{1}{1+\omega_{c} \Delta t}
\end{aligned}
$$

359

where $V_{f}$ is the complex MTI filtered output, $V_{u}$ is the complex unfiltered input, $n_{f t}$ is the $n_{f t}^{t h}$ sample along the slow time axis and $\omega_{c}$ is the filter angular cut-off frequency. The cut-off frequency is $\frac{1}{6 \Delta t}$ where $\Delta t$ is the pulse repetition interval, which is in this case $20 \mathrm{~ms}$. This cut-off frequency was chosen to satisfy the Nyquist frequency and as it was found to give the best separation of stationary clutter and moving snow. Note that the Equation (15) shows the filter in its low-pass form which isolates the stationary clutter. The moving snow can be isolated by taking the difference between the input data and the low-pass filtered data (thus behaving as a high-pass filter).

Following MTI processing, one can take an overall view of the avalanche by incoherently summing the output of the MTI filter from each receiver channel. The effect of this process is to reduce the power level of Gaussian noise corrupting the image by $9 \mathrm{~dB}$. This resultant image is equivalent to that given by a single channel range radar with a range-resolution of $1.3 \mathrm{~m}$. An example of such an image of an avalanche is shown later in this paper (Figure 10). From this image, velocity measurements averaged over the azimuthal beamwidth of a single antenna can be calculated. Indeed, velocity measurements from such images have been shown to agree well with in-situ avalanche measurements in our initial efforts to cross-validate the radar mea- 
surements with those obtained from sensors in the avalanche path (Vriend et al., 2013).

Now that we have corrected for array distortions, performed beamforming and MTI-filtering, it is trivial to generate 2-D images of the avalanche. The output of the processing methods is a 3-D array for each of the six chirps, the axes of which are slant range, pointing direction and time. The pointing direction can be converted to cross-range using a polar to Cartesian conversion, which gives an image that is more easily interpreted. The set of images can be animated to watch the progress of the avalanche in two dimensions. This is the first time we have been able to image the dense region of a flowing avalanche using a radar sensor. Examples of the 2-D images of an avalanche are shown later in this paper.

\section{Avalanche Test Site}

The radar is deployed at the Vallée de la Sionne (VDLS), Switzerland avalanche test site (Ammann, 1999). It is installed in a reinforced-concrete bunker facing a mountain face that is prone to avalanches. Here it takes non-invasive measurements of entire avalanche events throughout the winter season making use of an automated triggering system. The test site has two main avalanche tracks; Crêta Besse 1 (CB1) and Crêta Besse 2 (CB2).

The triggering system consists of a network of seismic sensors located in the avalanche track. When the seismic signal measured by these sensors exceeds a certain threshold it is assumed that an avalanche is in progress and the measurement instruments are switched on, including the new radar. Other sensors exist within the avalanche track including optical and pressure 
sensors which are housed on a mast located in the CB1 track at $\approx 660 \mathrm{~m}$ range.

The radar modules are located within the avalanche bunker to protect them from the weather. The antennas are set up on the outside walls of the bunker as shown in Figure 5. The transmitter antenna is located on the south-facing wall of the bunker, pointing towards the centre of the avalanche track $\left(12^{\circ}\right.$ relative to horizontal). The eight receiver antennas are spaced as per the array design (Figure 6), also pointing towards the centre of the avalanche track. The straight line distance between the transmitter and the closest receiver antenna of $\approx 1.8 \mathrm{~m}$, combined with the concrete wall, provides an isolation between the transmitter and receiver of approximately $70 \mathrm{~dB}$ (calculated from lab measurements combined with a conservative estimate of $10 \mathrm{~dB}$ loss across the concrete wall). A more conservative figure of $50 \mathrm{~dB}$ isolation was designed in to the system.

Loop tests were carried out following the installation of the radar to de-embed channel-to-channel transmission line length differences from the range measurements. In addition, a trihedral reflector was carried to different positions in the avalanche track and a measurement of the scene was made with the radar. A GPS coordinate was taken at each point. The locations of the reflector are plotted on a digital terrain model of the mountain in Figure 8. Following processing of the measured data, the 2-D locations of the reflector were recorded and used to calibrate the cross-range measurements of avalanches following full-array processing. This meant that the 2-D location of the avalanche could be plotted with confidence. 


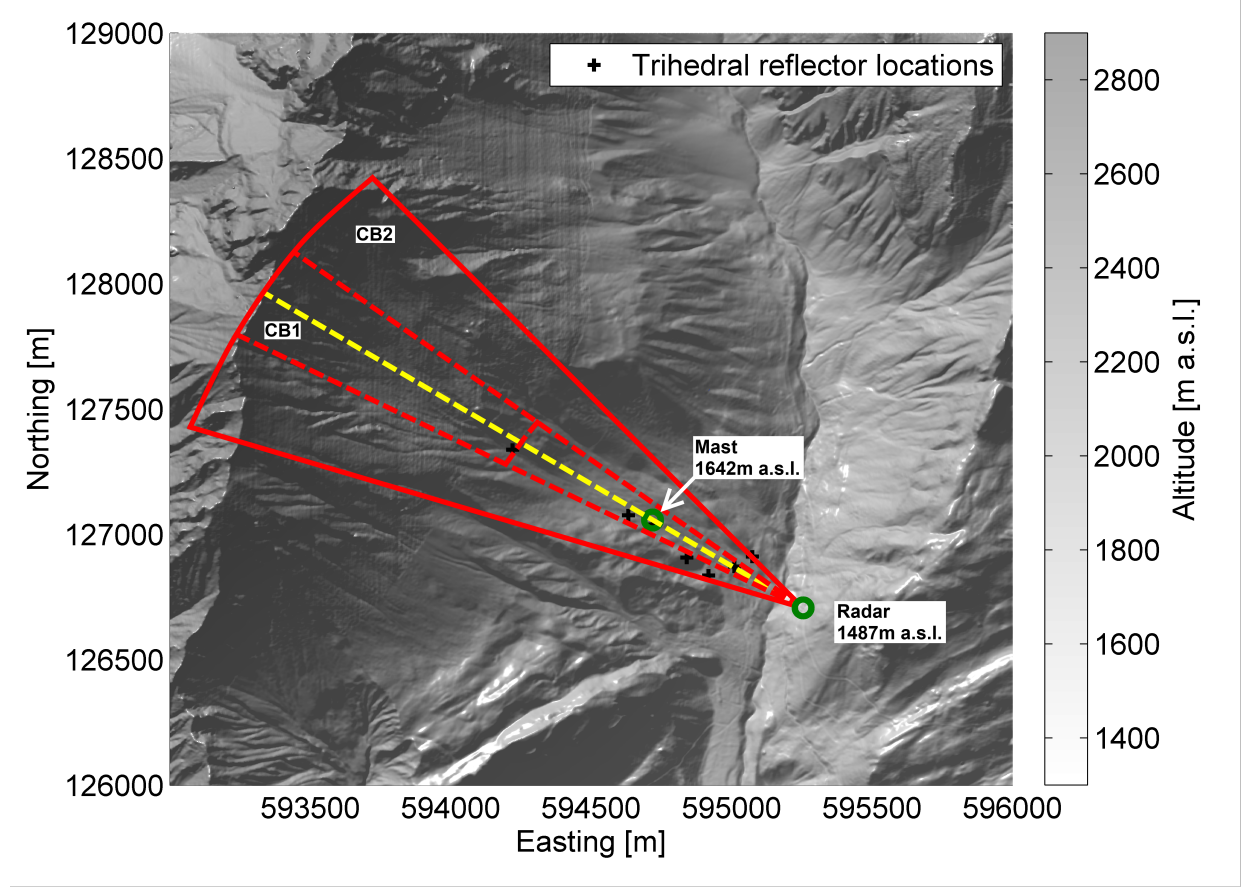

Figure 8: Radar, mast and trihedral target locations plotted on a digital terrain model of the Vallée de la Sionne (VDLS) site with the release zones of the two main avalanche tracks, Crêta Besse 1 (CB1) and Crêta Besse 2 (CB2), labelled. The area within the red lines indicates the azimuthal area within the half-power beamwidth of the radar antennas. The trihedral locations are within this area. The red dashed lines indicate $\pm 4.8^{\circ}$ relative to the radar-mast baseline (yellow dashed line), which equates to a cross-range of $100 \mathrm{~m}$ at $1200 \mathrm{~m}$ slant range from the radar. 


\section{Avalanche Observations}

The radar has been operational since the 2010 winter season and in this paper we are able to publish two-dimensional radar images of a flowing avalanche for the first time. Range and velocity measurements of the avalanche under discussion have been presented by Vriend et al. (2013).

Optical images of the avalanche track are taken every 30 minutes and these reveal its state before and after the avalanche examined in this paper took place (the avalanche occurred during the night). These images are shown in Figure 9. They reveal the scale of the avalanche, with much of the flow reaching well into the run-out zone of the avalanche track. It is clear that a portion of the flow reached the mast. Two regions have been highlighted which will be discussed during the analysis of the 2-D radar images. These two regions are clearly areas where movement has occurred and this is confirmed in the radar data. Other regions of movement have not been seen in the radar data to date but it is believed with further processing they can be revealed.

The range data were processed to produce range-time images of the data following MTI filtering. An example image can be seen in Figure 10. A similar image, complete with a detailed discussion of observations, is shown in Vriend et al. (2013). This range-time image was generated using the data from chirp 6 and the pixel intensity has been averaged over all eight channels of the receive array. The pixel intensity (squared magnitude of pixel following MTI) is normalised to the peak image intensity. It can be seen that the trigger occurred following the development of a significant avalanche front, hence the beginnings of the avalanche cannot be seen. The initial avalanche front is 


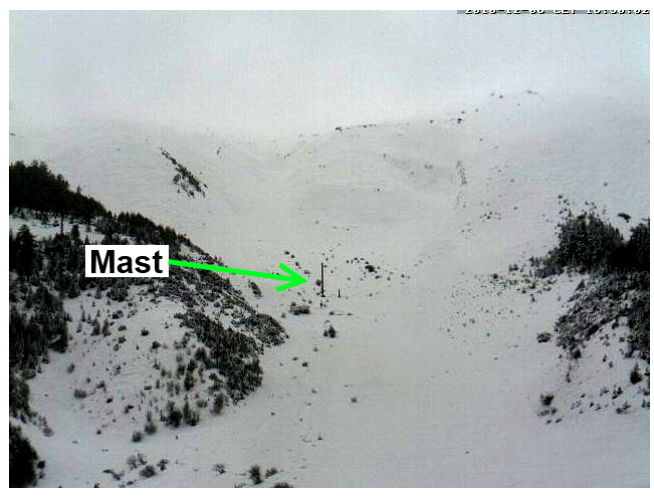

(a)

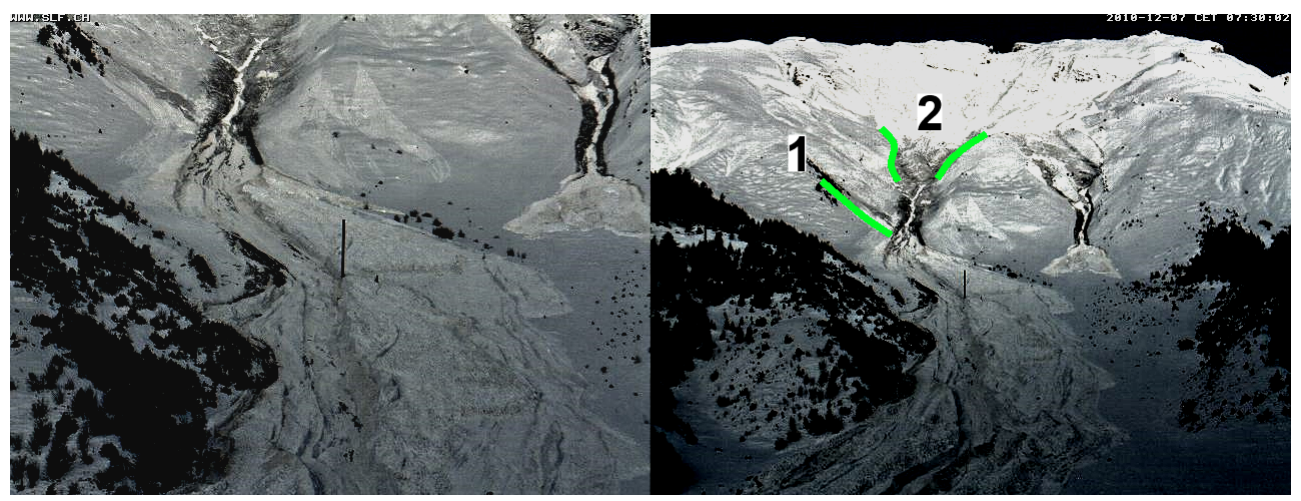

(b)

Figure 9: Optical images of avalanche track taken (a) before and (b) after two naturally occurring avalanches. Two regions of the flowing zone have been labelled. These will be referred to during analysis of the 2-D radar images. 
measured from when it reaches slant range $1200 \mathrm{~m}$ (the line-of-sight range from avalanche bunker). There are four significant avalanche fronts, which overlap over time, which have been labelled in the image. Additionally, it is clear that there is significant movement behind each front. One can follow individual streaks within the image, which are thought to be caused by the movement of a layer or particle along the path of the flow. Two 2-D images have been selected as a demonstration of the imaging capability of the radar. These are single frame snapshots of the avalanche. Two boxes in Figure 10 are centred on the time of snapshots for comparison.

The 2-D images are presented in polar space with the angle converted to an equivalent cross-range. The images are shown in Figures 11 and 12. They reveal the range and cross-range location of components of the avalanche flow. Figure 11 shows the first frame of the dataset. We can see that there are at least two avalanche fronts at this point in time (labelled Front 1a and Front 1b), each at a similar slant range but separated in cross-range. This information was not revealed by the single channel data and demonstrates the value of the receiver array design. An artefact at a cross-range of approximately $75 \mathrm{~m}$ in this image also shows an inherent weakness of the antenna array, that is the effect of the sidelobes discussed in Section 2.4. It may be possible to disregard these artefacts with further processing. The artefacts will always have a lower signal strength than the real avalanche response. Furthermore, they may appear in areas of the track where avalanches are simply unlikely to travel. The issue of artefacts from the antenna array will be improved in the second iteration of the radar which will be discussed later in this text. 


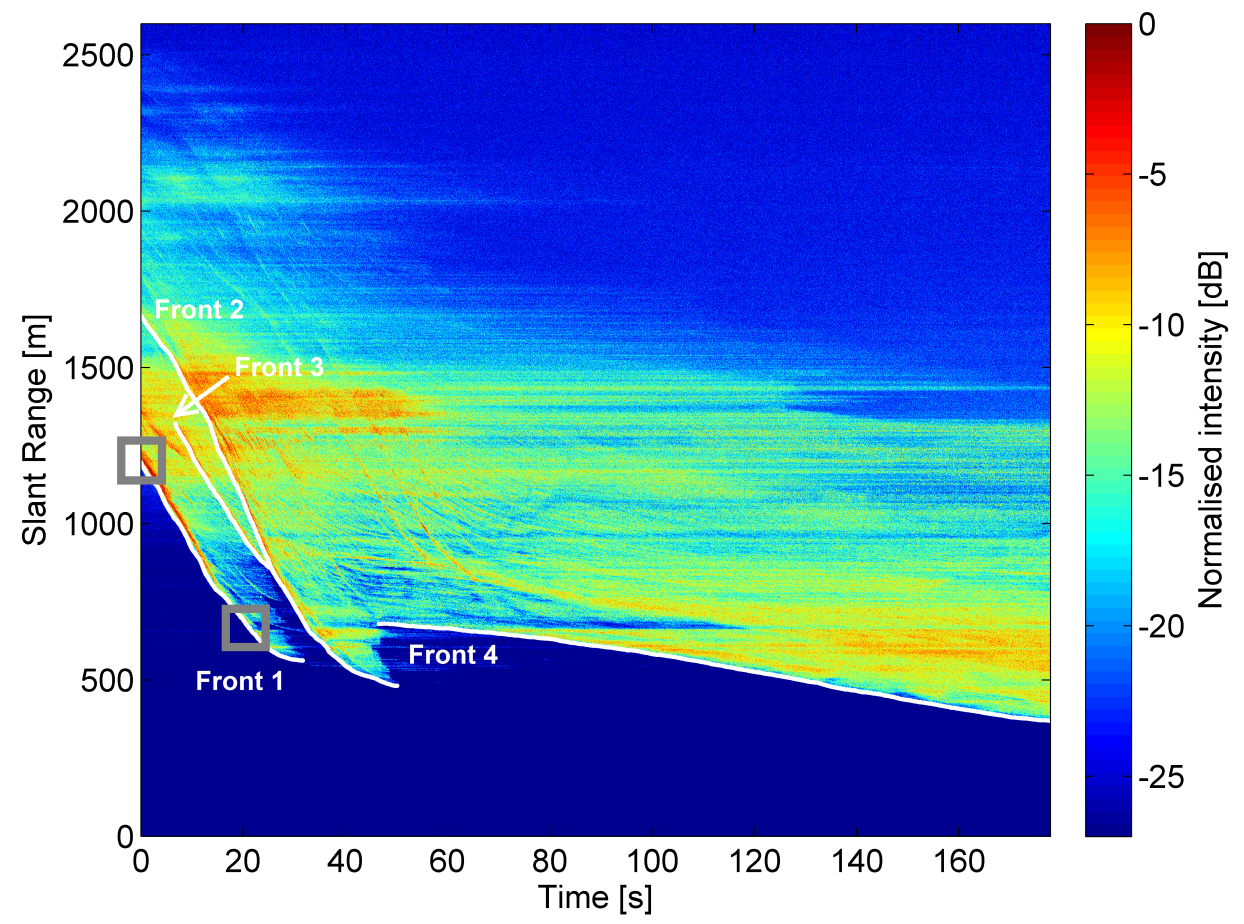

Figure 10: Range-time moving target indication (MTI) image of avalanche composed using an average of all eight receiver channels for the chirp 6 data. Four significant avalanche fronts have been labelled. The two boxes indicate the regions from which the following two 2-D images are taken. 
Figure 12 shows the moment when the first avalanche front meets the measurement mast. This image reveals a large number of strong reflectors within the avalanche, with a region of particularly high returns extending some $40 \mathrm{~m}$ in the cross-slope direction and approximately $70 \mathrm{~m}$ in the longitudinal direction. Clearly, the advantage of the radar in identifying and tracking features that never pass adjacent to the mast is clear. In addition, data of this resolution permit cross-correlation of such features between the radar and the mast and subsequent, pseudo-Lagrangian-based analysis, which is not possible with fixed-point instrumentation. Such analyses will form the basis for future research.

Using the single channel slant range measurements as a guide, the path taken by the avalanche was measured manually by recording the slant range and bearing of the peak return of each of the four avalanche fronts identified in Figure 10 at one second intervals. These recordings were then converted to the same geographic coordinate system as used for the digital terrain model shown in Figure 8. The result is plotted in Figure 13 . Avalanche front 1a was at a cross-range of $-70 \mathrm{~m}$ when the recording started. This region was highlighted in the optical image of the avalanche track following the two avalanches (Figure 9 label 1). At this point in time, there were two avalanche fronts present, which cannot be seen in the single channel data. They are only revealed in the 2-D data as labelled in Figure 11. Front 1b appears at a cross-range of $8.5 \mathrm{~m}$ which corresponds with a gully (Figure 9 label 2). Hence, the avalanche is believed to have originated in Crêta Besse 1 with a secondary flow (front 1a) feeding in from the left of the gully. Analysis of the 2-D images reveals that the secondary flow collides with front $1 \mathrm{~b}$ at 


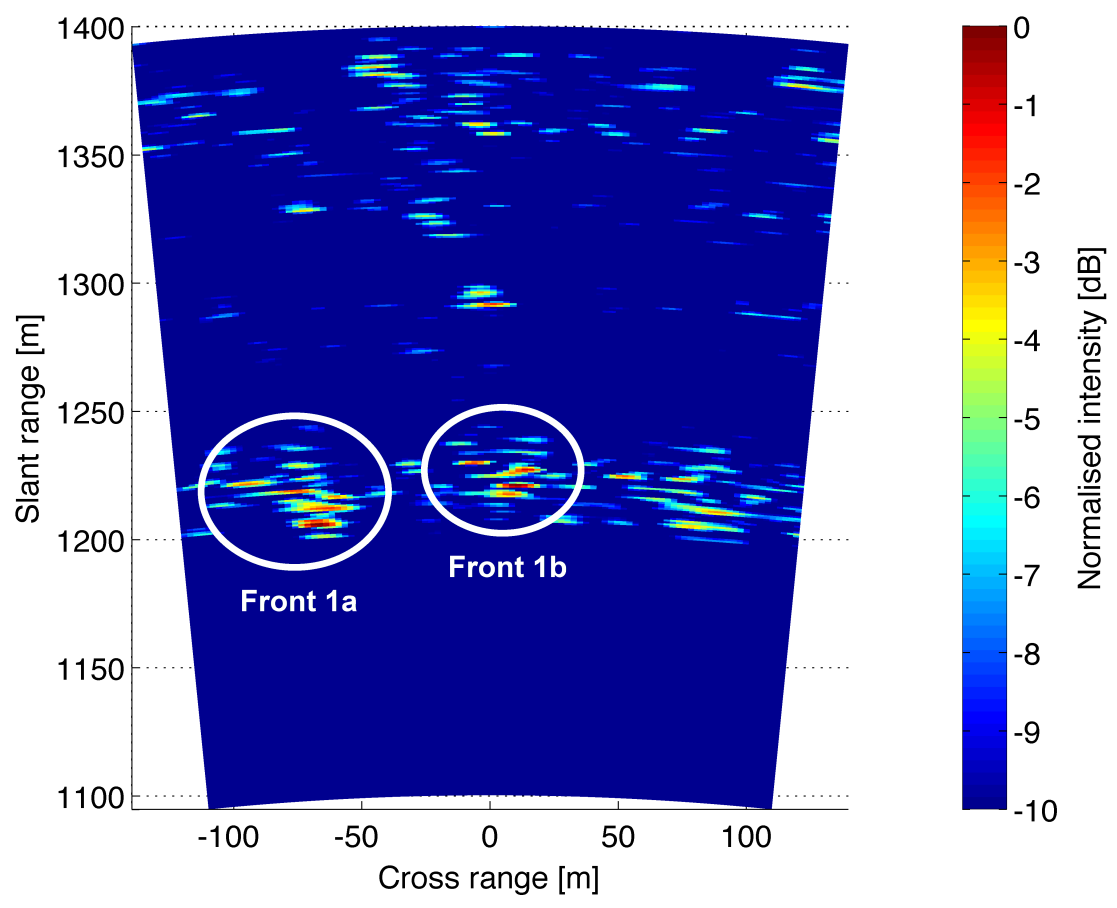

Figure 11: 2-D image of avalanche at $T=0.00 \mathrm{~s}$ using chirp 6 . Image shows the presence of two fronts (circled) separated in cross-range but very similar in slant range. The artefact at a similar slant range, but $75 \mathrm{~m}$ cross-range, is caused by the sidelobes of the antenna array discussed in Section 2.4 . 


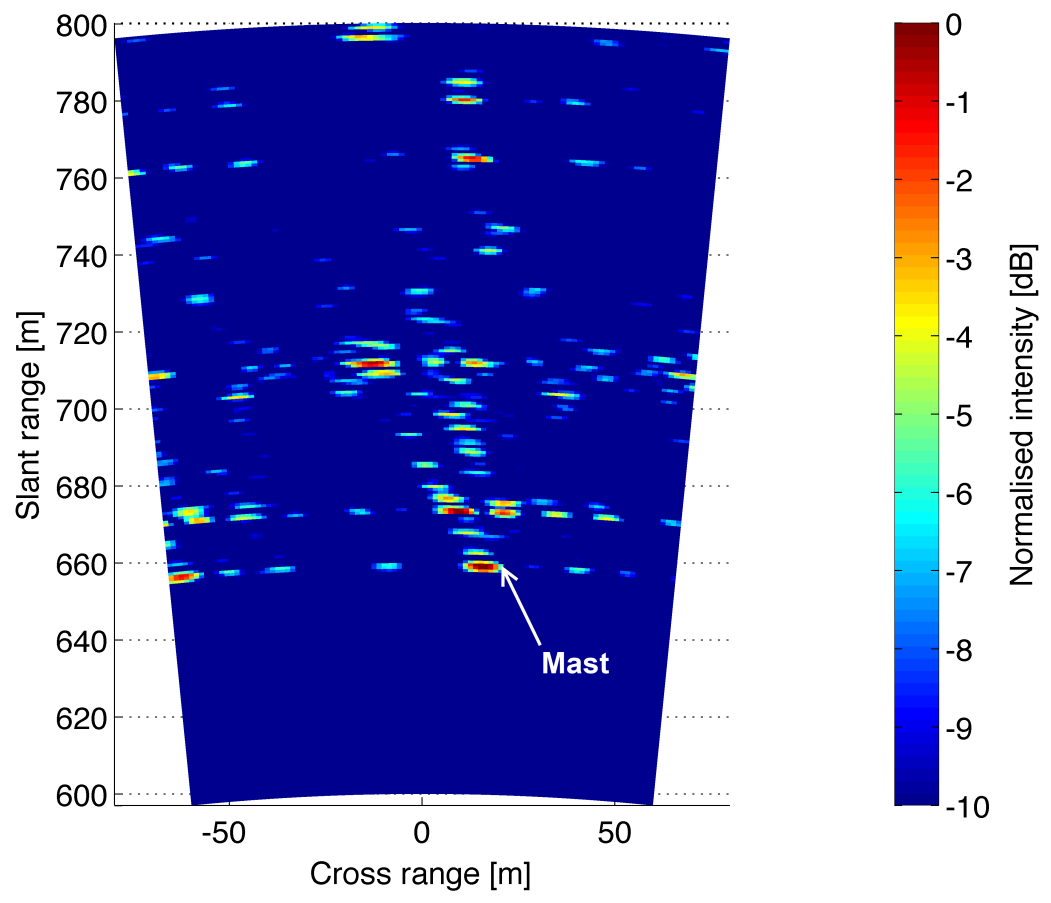

Figure 12: 2-D image of avalanche at $T=21.96 \mathrm{~s}$ using chirp 5 . This shows a snapshot of the avalanche meeting the measurement mast at around $660 \mathrm{~m}$ range. The image also shows the presence of many components of the avalanche. 
approximately $1106 \mathrm{~m}$ range. Following this, the three trailing fronts seem to follow a similar path in to the run-out zone. However, the fronts do stop at different slant ranges. This shows the potential for using the sensor data to study the effect of recently deposited snow on the run out distances of trailing fronts. With tracking of the avalanches front, periods and regions of interest during the avalanche can be pinpointed and the two-dimensional images can be studied in greater detail, such as looking at the interactions of various components of the avalanche. This targeted approach is necessary due to the extreme wealth of data collected during a single avalanche (50 high-resolution images per second). Indeed, it will take some time to realise the full potential of the data generated by this new radar. 
(b)

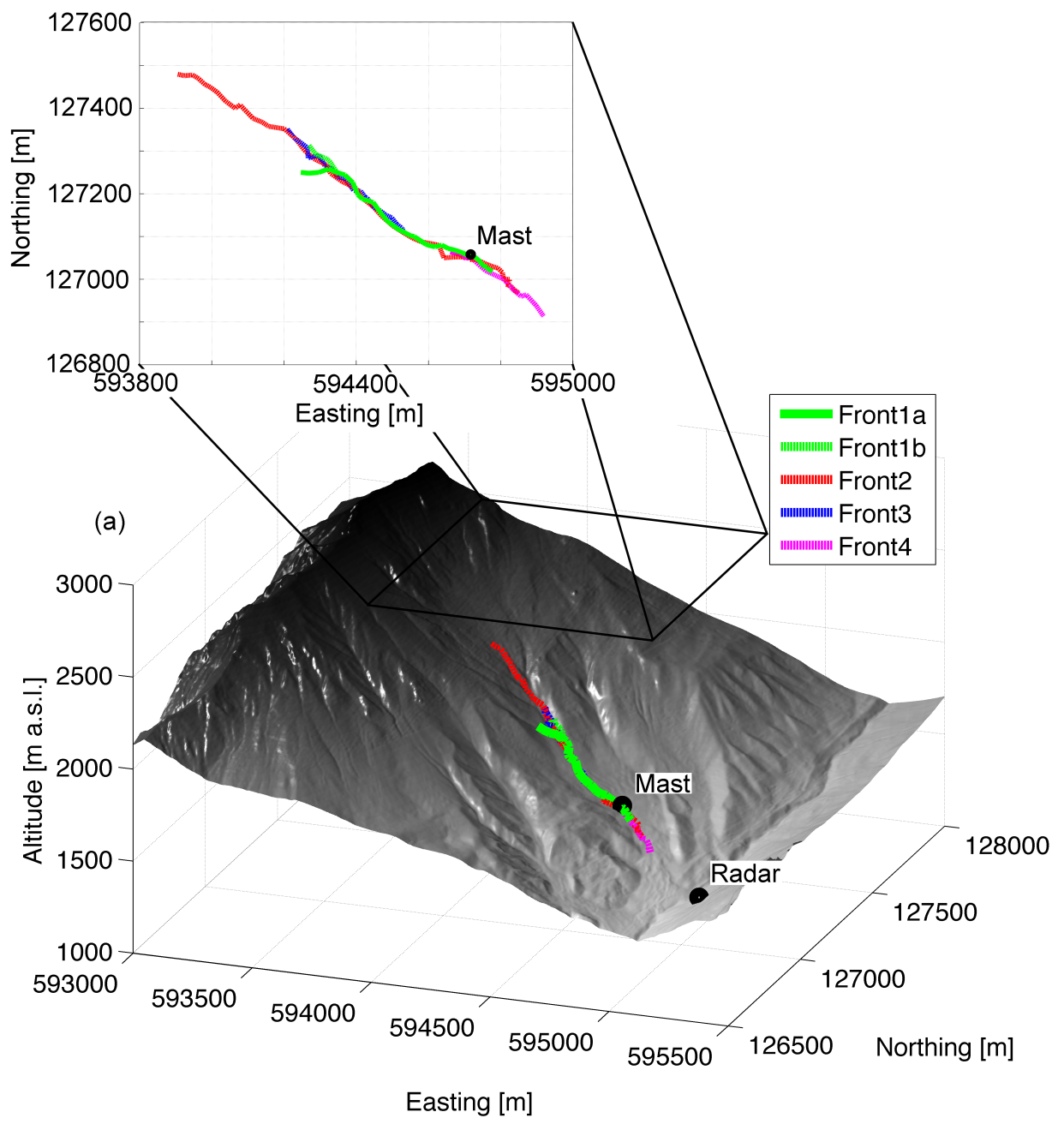

Figure 13: (a) Path taken by avalanche fronts as recorded by the radar plotted on a topographical map of the Vallée de la Sionne site (generated using the digital terrain model) and (b) top-down view of paths plotted in a geographical coordinate system. The paths were measured manually by recording the slant range and bearing of the peak return of each of the four avalanche fronts at one second intervals and then converting these measurements to a geographical coordinate system. 


\section{Conclusions and Future Work}

Avalanches have been measured using remote sensing for some time now, however existing sensors have clear weaknesses. It was concluded that the data collected by radar sensors in particular could be improved to complement existing measurements for validating mathematical models of avalanches. This paper has described the hardware and signal processing design of a radar for measuring avalanches that outperforms the existing radar sensors in terms of range resolution, and also provides cross-range resolution to produce twodimensional radar images of flowing avalanches for the first time.

A series of images of a recorded avalanche have been presented in this paper. These images show the flowing avalanche in unparalleled detail. Both the range-time and 2-D images reveal what are thought to be the movements of layers or particles along the path of the avalanche. We have also been able to manually track the avalanche in two spatial dimensions.

The sensor has been reliably collecting data for a few winter seasons now. The processing of the collected data is a demanding task and the processing techniques are always being refined. In the future, we would like to look towards automated tracking of avalanche components over time in two spatial dimensions. Using this tracking information we can start to generate velocity maps of the dense flowing part of avalanches from a significant library of measurement data. To produce accurate velocity measurements, the range and velocity information embedded in the beat signal (Equation (6)) needs to be resolved. This processing should make use of the data from each of the six chirps (Figure 2). The velocity measurements can be validated against velocity data measured by other instruments within the avalanche 
track (Tiefenbacher and Kern, 2004, Gubler, 1986). It is believed this will provide a valuable database for researchers wanting to validate models for avalanche dynamics. For example, while traditional block models (Perla et al., 1980) and certain shallow-water based models are implemented for flow down a slope profile, it is clearly more realistic to make use of models that allow the flow to evolve in the cross-slope direction Naaim et al. 2004; Sampl and Zwinger, 2004; Christen et al., 2010). However, this has implications for friction parameterisation (Barbolini et al., 2000) and with a dearth of data to validate such codes, it has not always been clear that physical realism outweighs increased uncertainty in parameterisation. The great benefit of our work is that proper validation and calibration of these models becomes possible. Furthermore, with our data, combined with field measurements over a number of avalanches of antecedent snow conditions, we have the potential to form and calibrate a mixture model for the density (i.e. the balance of wet and dry snow) that can then be used to enhance the parameterisation of numerical models of avalanche dynamics.

Given the demonstrable success of the sensor both in terms of validated velocity information (Vriend et al., 2013) and the cross-range processing shown here, we are already developing an improved version with a 16-element receiver antenna array. This hardware development will give marked improvement in the quality of the cross-range measurements of avalanches and further improve the sensitivity of the receivers. 


\section{Acknowledgements}

The authors would like to thank the Swiss Federal Institute of Snow and Avalanche Research for their help throughout the duration of this work. In particular, we would like to acknowledge the continued support of Martin Hiller and François Dufour. The authors are grateful to the Natural Environment Research Council for their support, grant ref. NE/F004621/1, and to the Engineering and Physical Sciences Research Council for allowing us to continue this work with their support, grant ref. EP/K00767X/1. Finally, we would like to thank Dr. Lai Bun Lok for his valuable review of this paper, and the journal reviewers for their helpful comments and suggestions.

\section{References}

Ammann, W.J., 1999. A new swiss test-site for avalanche experiments in the Vallée de la Sionne/Valais. Cold Regions Science and Technology 30, $3-11$.

Ancey, C., Meunier, M., 2004. Estimating bulk rheological properties of flowing snow avalanches from field data. Journal of Geophysical Research: Earth Surface 109, F01004.

Barbolini, M., Gruber, U., Keylock, C., Naaim, M., Savi, F., 2000. Application of statistical and hydraulic-continuum dense-snow avalanche models to five real european sites. Cold Regions Science and Technology 31, 133149.

Bertrand, D., Naaim, M., Brun, M., 2010. Physical vulnerability of reinforced 
concrete buildings impacted by snow avalanches. Natural Hazards and Earth System Science 10, 1531-1545.

Blanchet, J., Marty, C., Lehning, M., 2009. Extreme value statistics of snowfall in the Swiss Alpine region. Water Resources Research 45, W05424.

Bouchet, A., Naaim, M., Ousset, F., Bellot, H., Cauvard, D., 2003. Experimental Determination of Constitutive Equations for Dense and Dry Avalanches: Presentation of the Set-Up and First Results. Surveys in Geophysics 24, 525-541.

Christen, M., Kowalski, J., Bartelt, P., 2010. RAMMS: Numerical simulation of dense snow avalanches in three-dimensional terrain. Cold Regions Science and Technology 63, 1-14.

Dent, J.D., Burrell, K.J., Schmidt, D.S., Louge, M.Y., Adams, E.E., Jazbutis, T.G., 1998. Density, velocity and friction measurements in a dry-snow avalanche. Annals of Glaciology 26, 247-252.

Eckert, N., Naaim, M., Parent, E., 2010. Long-term avalanche hazard assessment with a bayesian depth-averaged propagation model. Journal of Glaciology 56, 563-586.

Eglit, M.E., 1968. Theoretical approaches to the calculation of the motion of snow avalanches. Itogi Nauki, Moscow, VINITI , 60-97. English translation in Glaciological Data, Report GD16 (1974).

Friis, H., 1944. Noise Figures of Radio Receivers. Proceedings of the IRE 32, 419-422. 
Gauer, P., Issler, D., 2004. Possible erosion mechanisms in snow avalanches. Annals of Glaciology 38, 384-392.

Gauer, P., Kern, M., Kristensen, K., Lied, K., Rammer, L., Schreiber, H., 2007. On pulsed Doppler radar measurements of avalanches and their implication to avalanche dynamics. Cold Regions Science and Technology $50,55-71$.

Gray, J.M.N.T., Tai, Y.C., 1998. On the inclusion of a velocity-dependent basal drag in avalanche models. Annals of Glaciology 26, 277-280.

Gubler, H., 1986. Measurements and modelling of snow avalanche speeds, in: Avalanche Formation, Movement and Effects: Proceedings of the Davos Symposium.

Gubler, H., Hiller, M., 1984. The use of microwave FMCW radar in snow and avalanche research. Cold Regions Science and Technology 9, 109-119.

Gubler, H., Hiller, M., Klausegger, G., Suter, U., 1986. Messungen an Fliesslawinen, Zwischenbericht 1986. Eidg. Institut für Schnee- und Lawinenforschung, Weissfluhjoch, Switzerland, 41.

Hallikainen, M., Ulaby, F., Abdelrazik, M., 1986. Dielectric properties of snow in the 3 to $37 \mathrm{GHz}$ range. IEEE Transactions on Antennas and Propagation 34, 1329-1340.

Harris, F.J., 1978. On the use of windows for harmonic analysis with the discrete Fourier transform. Proceedings of the IEEE 66, 51-83. 
Issler, D., Harbitz, C.B., Kristensen, K., Lied, K., Moe, A.S., Barbolini, M., De Blasio, F.V., Khazaradze, G., McElwaine, J.N., Mears, A.I., Naaim, M., Sailer, R., 2005. A comparison of avalanche models with data from dry-snow avalanches at Ryggfonn, Norway., in: Senneset, K., Flaate, K., Larsen, J.O. (Eds.), Proceedings of the 11th International Conference and Field Trip on Landslides, Norway, Taylor \& Francis Group, Netherlands.

Kern, M., Tiefenbacher, F., McElwaine, J., 2004. The rheology of snow in large chute flows. Cold Regions Science and Technology 39, 181-192.

Keylock, C.J., Barbolini, M., 2001. Snow avalanche impact pressure/vulnerability relations for use in risk assessment. Canadian Geotechnical Journal 38, 227-238.

Keylock, C.J., McClung, D.M., Magnusson, M.M., 1999. Avalanche risk mapping by simulation. Journal of Glaciology 45, 303-314.

McClung, D., Schaerer, P., 2006. The Avalanche Handbook. third ed., The Mountaineers Books.

Naaim, M., Naaim-Bouvet, F., Faug, T., Bouchet, A., 2004. Dense snow avalanche modeling: flow, erosion, deposition and obstacle effects. Cold Regions Science and Technology 39, 193-204.

Nishimura, K., Maeno, N., 1988. Contribution of viscous forces to avalanche dynamics. Annals of Glaciology 13, 202-206.

Norem, H., Irgens, F., Schieldrop, B., 1986. A continuum model for calculating snow avalanche velocities, in: IAHS Publ. 162 (Symposium at Davos, 1986. Avalanche formation, movement and effects), pp. 363-379. 
Perla, R., Cheng, T.T., McClung, D.M., 1980. A two-parameter model of snow avalanche motion. Journal of Glaciology 26, 197-207.

Pozar, D.M., 2012. Microwave Engineering. fourth ed., John Wiley \& Sons.

Rammer, L., Kern, M., Gruber, U., Tiefenbacher, F., 2007. Comparison of avalanche-velocity measurements by means of pulsed Doppler radar, continuous wave radar and optical methods. Cold Regions Science and Technology 50, 35-54.

Rignot, E., Echelmeyer, K., Krabill, W., 2001. Penetration depth of interferometric synthetic-aperture radar signals in snow and ice. Geophysical Research Letters 28, 3501-3504.

Salm, B., 1993. Flow, flow transition and runout distances of flowing avalanches. Annals of Glaciology 18, 221-226.

Sampl, P., Zwinger, T., 2004. Avalanche simulation with SAMOS. Annals of Glaciology 38, 393-398.

Schaer, M., Issler, D., 2001. Particle densities, velocities and size distributions in large avalanches from impact-sensor measurements. Annals of Glaciology 32, 321-327.

Schreiber, H., 2001. Avalanche dynamics measurement by pulsed Doppler Radar. Annals of Glaciology 32, 275-280.

Skolnik, M., 2001. Introduction to Radar systems. third ed., McGraw-Hill.

Steinberg, B., 1976. Principles of Aperture and Array System Design. John Wiley \& Sons. 
Steinberg, B., 1981. Radar imaging from a distorted array: The radio camera algorithm and experiments. IEEE Transactions on Antennas and Propagation 29, 740-748.

Steinkogler, W., Sovilla, B., Lehning, M., 2014. Influence of snow cover properties on avalanche dynamics. Cold Regions Science and Technology 97, 121-131.

Stove, A., 1992. Linear FMCW radar techniques. IEE Proceedings For Radar and Signal Processing 139, 343-350.

Tiefenbacher, F., Kern, M., 2004. Experimental devices to determine snow avalanche basal friction and velocity profiles. Cold Regions Science and Technology 38, 17-30.

Tiuri, M., Sihvola, A., Nyfors, E., Hallikaiken, M., 1984. The complex dielectric constant of snow at microwave frequencies. IEEE Journal of Oceanic Engineering 9, 377-382.

Trees, H.L.V., 2002. Part IV of Detection, Estimation, and Modulation Theory: Optimum Array Processing. John Wiley \& Sons.

Ulaby, F.T., Moore, R.K., Fung, A.K., 1982. Microwave Remote Sensing: Active and Passive, Volume II: Radar Remote Sensing and Surface Scattering and Emission Theory. Addison-Wesley.

Ulaby, F.T., Moore, R.K., Fung, A.K., 1986. Microwave Remote Sensing: Active and Passive, Volume III: From Theory to Applications. AddisonWesley. 
Vriend, N.M., McElwaine, J.N., Sovilla, B., Keylock, C.J., Ash, M., Brennan, P.V., 2013. High-resolution radar measurements of snow avalanches. Geophysical Research Letters 40, 727-731.

\section{Figure Captions}

- Figure 1 (color): The effect of grazing angle $\psi$ on length measured along a surface and the effect of range on the azimuthal width of resolution cell.

- Figure 2 (color): Format of the system frequency modulated signal frames. The total frame duration is $20 \mathrm{~ms}$.

- Figure 3 (color): Full system block diagram of the frequency modulated continuous wave radar.

- Figure 4 (color): Predicted signal-to-noise ratio (SNR) for $5 \mathrm{~ms}$ up chirp using receiver noise measurements and a modelled target with a backscatter coefficient of 0.002 at $7^{\circ}$ grazing angle. The SNR at the maximum required range $(\approx 2500 \mathrm{~m})$ is over $18 \mathrm{~dB}$ which makes the system suitable for the detection of snow along the entire Vallée de la Sionne avalanche track.

- Figure 5 (color): Antenna array setup in Vallée de la Sionne test bunker. The transmitter antenna (TX) is located on the south-facing wall of the shelter pointing towards avalanche track at an angle of $12^{\circ}$ relative to horizontal. The eight receiver antennas (RX1-8) are randomly spaced 
above the four shelter windows. The receiver antennas are also pointed towards the avalanche track.

- Figure 6 (color): (a) Diagram of the linear receiver array antenna element positions used in the newly developed avalanche radar. The element locations have been randomised to minimise the effect of sidelobes. (b) Array factor of radar receiver array using directive cosine elements over $\sin \theta= \pm 0.26$ with array factor of uniformly spaced linear array with the same baseline and directive elements.

- Figure 7 (color): Example time domain data labelled into the returns from each of the six chirps. The data are reshaped into six twodimensional arrays with columns of short time and rows of fast time.

- Figure 8 (color): Radar, mast and trihedral target locations plotted on a digital terrain model of the Vallée de la Sionne (VDLS) site with the release zones of the two main avalanche tracks, Crêta Besse 1 (CB1) and Crêta Besse 2 (CB2), labelled. The area within the red lines indicates the azimuthal area within the half-power beamwidth of the radar antennas. The trihedral locations are within this area. The red dashed lines indicate $\pm 4.8^{\circ}$ relative to the radar-mast baseline (yellow dashed line), which equates to a cross-range of $100 \mathrm{~m}$ at $1200 \mathrm{~m}$ slant range from the radar.

- Figure 9 (color): Optical images of avalanche track taken (a) before and (b) after two naturally occurring avalanches. Two regions of the flowing zone have been labelled. These will be referred to during analysis of the 2 -D radar images. 
- Figure 10 (color): Range-time moving target indication (MTI) image of avalanche composed using an average of all eight receiver channels for the chirp 6 data. Four significant avalanche fronts have been labelled. The two boxes indicate the regions from which the following two 2-D images are taken.

- Figure 11 (color): 2-D image of avalanche at $T=0.00 \mathrm{~s}$ using chirp 6. Image shows the presence of two fronts (circled) separated in crossrange but very similar in slant range. The artefact at a similar slant range, but $75 \mathrm{~m}$ cross-range, is caused by the sidelobes of the antenna array discussed in Section 2.4.

- Figure 12 (color): 2-D image of avalanche at $T=21.96$ s using chirp 5. This shows a snapshot of the avalanche meeting the measurement mast at around $660 \mathrm{~m}$ range. The image also shows the presence of many components of the avalanche.

- Figure 13 (color): (a) Path taken by avalanche fronts as recorded by the radar plotted on a topographical map of the Vallée de la Sionne site (generated using the digital terrain model) and (b) top-down view of paths plotted in a geographical coordinate system. The paths were measured manually by recording the slant range and bearing of the peak return of each of the four avalanche fronts at one second intervals and then converting these measurements to a geographical coordinate system. 